\title{
Microstrip Stopband Split Ring Resonator for Microwave Microfluidic Sensing
}

\author{
Ahmed Adnan Al-Mudhafar * a, Ali A. Abduljabar ${ }^{b}$, and Hayder Jawad Albattat ${ }^{a}$ \\ ${ }^{a}$ Department of Communication Technologies Engineering, Engineering Technical College, University of Al-Furat Al-Awsat Technical, 31001 Najaf, Iraq. \\ ${ }^{b}$ Department of Electrical Engineering, College of Engineering, University of Basrah, 61001 Basrah, Iraq
}

\section{ART ICLE INFO}

\section{Article history:}

Received 17 March 2020

Received in revised form 25 April 2020

Accepted 27 April 2020

\section{Keywords:}

Split ring resonator

Complex permittivity

Microfluidic Chanel

Sensitivity

\begin{abstract}
A B S T R A C T
Stopband split ring resonator (SSRR) is presented in this paper for measuring the complex permittivity of liquid with different positions of microfluidic channel operates at a resonant frequency of $1 \mathrm{GHz}$. The sensor was fabricated and microfluidic channel is located in the gap groove with two different positions of the carrier where the electric field is as large as possible. The sensor has been tested with several solvents to verify its sensitivity where the electric field interacts with the liquid filled in a quartz tube and hence alter the SRR behavior. The electromagnetic properties (complex permittivity) of the solvent can be extracted from the shift in the resonant frequency of the resonator due to the perturbation phenomenon.
\end{abstract}

(C)2020 University of Al-Qadisiyah. All rights reserved.

\section{Introduction}

Dielectric loss and dielectric constant of the materials are both of which are functions of many parameters that are the field's frequency, temperature, pressure, orientation, mixture, and molecular structure of the material [1]. Many methods have been used to measure the material properties among these resonator resonant techniques offer numerous advantage compared to traditional methods resulting inaccurate measuring of material characterization to get maximum sensitivity the sample inserted posited in a place where the electric field is as large as a possible result shift resonant frequency and peak attenuation due to perturbation of sensor[2, 3].

In recent years, researchers have placed liquid under test in a region where the electric field is the greatest possible. Some of them have put the tube in contact with the upper surface of the sensor, inserted the tube inside substrate of the microstrip sensor, placed the tube non-contact with the sensor, and some of them have put the capillary between the substrate and ground plate of the sensor [4-9]. All of them are looking for a more sensitive and accurate measurement of complex permittivity of material /liquids for many applications is required in medicine and pharmaceuticals, surface crack, food industry, and chemical.

In this study, design and fabrication of the stopband microstrip split ring resonator is proposed as a microfluidic sensor. A cylindrical aluminum cavity is fabricated to enhance the sensitivity where the quality factor has been increased. Two positions of the microfluidic tube have been presented to study of the effect of the electric field direction

* Corresponding author.

E-mail address: ahmedadnan_ahmedadan@yahoo.com(Ahmed Adnan Al-Mudhafar) 


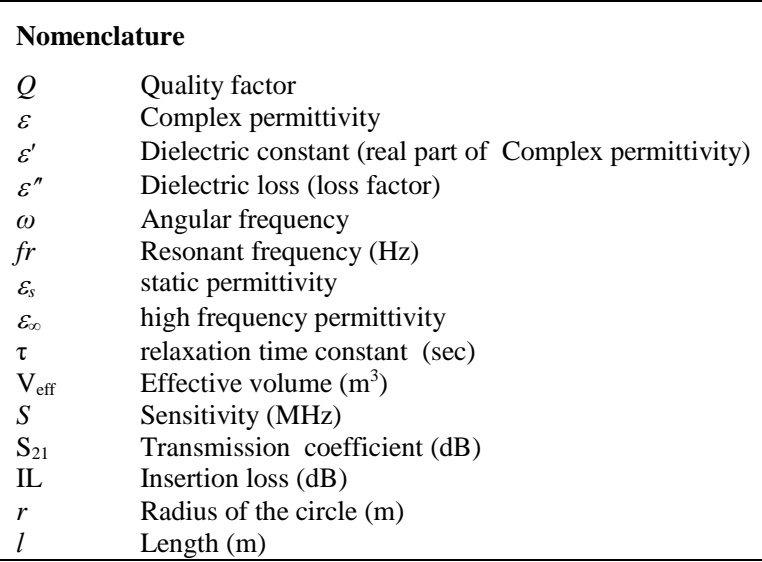

on the liquid sample. The sensor has been tested with several solvents to verify its sensitivity. The complex permittivity of the solvents have been computed from a shift in the resonant frequency of the resonator where the electric field interaction with the volume of the liquid filled.

\section{Theory And Concept}

\subsection{Basic Principle of SRR and frequency mode}

To understand the basic phenomena underlying the operation of the split ring resonator, it is first necessary to understand when resonance occurs and understand its electric field configuration for the different frequency modes.

Resonance The complex permittivity of the solvents have been computed when standing waves are set up in a $\lambda / 2$ open-circuited microstrip SRR [10]; this happens when magnetic energy stored in ring loop equal to the electric energy stored in the gap[11].

Single open end of a microstrip transmission line surrounds $70 \%$ of the SRR as a result tightly coupled used in order to achieve maximum power transfer. Shows the capacitive coupling suffered from high crosstalk and inductive coupling far less susceptible to crosstalk [12], so the symmetric feed lines are positioning at a magnetic field antinode were used to excite the split ring by inductive couplings with a smaller degree of capacitive coupling [3,13] as depicted in the Fig.1. Electric field of the SRR configuration for the different modes where symmetric microstrip lines are in parallel with a separation distance as shown in Fig.1. This line support two absolute value of maximum field point for the first two quasi-TEM modes of split ring, which are the odd and even modes as shown in the Fig.1.(a) even mode it can be seen the positive maximum electric field point $(+)$ at both side of the slit (same voltage sign) even mode capacitance, the absence electric field on the liquid so this capacitance neglected [5]. Fig.1.(b) odd mode it can be seen the positive maximum electric field point $(+)$ and negative maximum electric field point (-) at gap (opposite voltage sign) resulting from odd mode capacitance [14], any significant effect on frequency resonator could be a reflection of decrease or increase this capacitance due to variation in the electrical length of ring resonator so odd mode, Within the framework of this research, high sensitivity because perturbed by dielectric properties of the liquid within the gap.

$\begin{array}{ll}x & \text { X-coordinates }(\mathrm{m}) \\ y & \text { Y-coordinates }(\mathrm{m}) \\ \text { Greek symbols } \\ \sigma & \text { conductivity of copper }(\mathrm{S} / \mathrm{m}) \\ \lambda & \text { wavelength }(\mathrm{m}) \\ \Omega & \text { Omega unit of electrical resistance } \\ { }^{\circ} \mathrm{C} & \text { Celsius temperature } \\ \varnothing & \text { Angle (degree) } \\ \text { Subscripts } & \\ \Delta & \text { Change or shift } \\ \text { VNA } & \text { Vector network analyzer } \\ \text { SMA } & \text { Sub-Miniature version A connector }\end{array}$

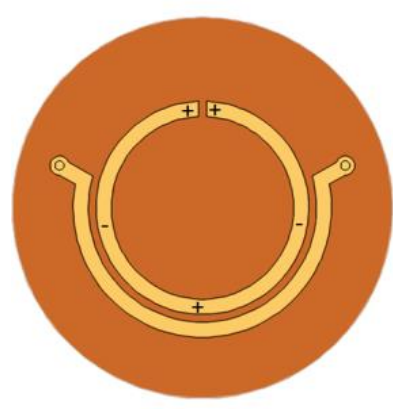

(a)

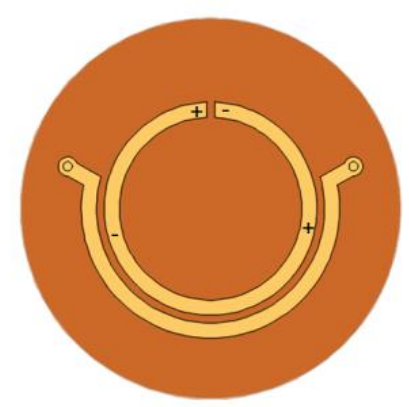

(b)
Figure 1. Maximum field point for the first two points :(a) even mode, (b) odd mode.

\subsection{Perturbation Theory and liquid complex permittivity}

Slit perturbation resonator arises when the slit is made in the microstrip ring resonator and maximum electric field at both the slit edge $[3,14]$ so one of its applications is determining complex permittivity of a sample by measuring the shift in frequency resonator bandwidth, insertion loss, and the quality factor (a degree of the loss of a resonant circuit lower loss infers a higher $\mathrm{Q}$ ) when a sample is inserted posited in a place where the electric field is as large as possible into a resonator[5] $[10,15]$.

Under the action of an external electric field on the atoms and molecules of liquid will exercise torque on the electric dipole the dipole will absorb microwave energy and rotate to align with the electric field at a time (called relaxation time constant) hence the positive particles are pushed in the direction of the field while the negative particles in the opposite direction from their equilibrium position causing dipole polarization. As a result of friction force between adjacent dipoles as they rotate toward the electric field, the absorbed energy is converted to heat $[3,16,17]$. Relative permittivity of a large number of liquid is a complex number and is expressed as follows [15-20]:

$\varepsilon=\varepsilon^{\prime}-\mathbf{j} \varepsilon^{\prime \prime}$

Complex dielectric constant as a function of frequency derived by Debye theory are given by $[19,20]$ :

$\varepsilon^{\prime}=\varepsilon_{\infty}+\frac{\varepsilon_{s}-\varepsilon_{\infty}}{1+(\omega \tau)^{2}}$ 
$\varepsilon^{\prime \prime}=\frac{\left(\varepsilon_{s}-\varepsilon_{\infty}\right) \omega \tau}{1+(\omega \tau)^{2}}$

where $\varepsilon^{\prime}$ corresponds to the real part, $\varepsilon^{\prime \prime}$ to the imaginary part, $\omega$ $=2 \pi \mathrm{f}, \tau$ is relaxation time constant, $\varepsilon_{S}$ is the static permittivity $(\mathrm{f}=0)$, and $\varepsilon_{\infty}$ is the high frequency permittivity $(\mathrm{f}=\infty)$. The real part of the permittivity, or dielectric constant, is the reactive part of the permittivity, that is, it represents a relative measure of the electromagnetic energy density that is stored inside the material and an imaginary part influences energy absorption is known as a "loss factor" and is a measure of how dissipative or lossy a material is to an external electric field [1].

\subsection{Microfluidic positions and Sensitivity}

In the previous sections, the electric field is concentrated as maximum as possible at the gap of the annular Ring (odd mode capacitance), which allows the position of the microfluidic channel in that gap vertically or the horizontal position as described in the Fig.2. And when the fluid-filled in the tube is placed in that channel this affects SRR behavior in terms of resonance frequency and losses which have been used to extract the values of the complex permittivity of the solvent. The effect of that capacitor is a variation for both tube states, but its effect is greater on the resonant properties of the sensor when edge parallel plate capacitor is filled with high dielectric liquid constant[21] but a little shift in the frequency of the SRR because more Large volume of the liquid exposed to the electric field so can be written that as [3] :

$$
\Delta f r \alpha \frac{1}{V e f f}
$$

The sensitivity of the sensor increases when there is a noticeable change in its resonance frequency which is expressed by the equation [6]:

$$
S=\frac{\Delta f r}{\Delta \varepsilon^{\prime}}
$$

\section{Fabrication of The Sensor and Micro-fluidic System}

Fig.3. Photograph of the fabricated SRR and $50 \Omega$ microstrip transmission line on PCB with dielectric constant substrate FR4 (dielectric thickness is $1.5 \mathrm{~mm}$, relative permittivity of $4.6 \pm 0.02$, and loss tangent 0.017) and dual conductive copper surfaces (thickness of the copper is $35 \mu \mathrm{m}, \sigma$ is $2.7 \times 107 \mathrm{~S} / \mathrm{m}$ ). SMA connectors with $50 \Omega$ used as input and output port of the resonator. General schematics of the SRR are presented in Fig.4. and Table 1 respectively.

Maximum electric field distribution is at the edge of ring gap so the horizontal channel (width is $1 \mathrm{~mm}$, and depth is $0.5 \mathrm{~mm}$ ) was made on the microstrip upper surface from the center to the circumference of the circle passing through the ring gap and vertical channel is made a circular hole (diameter is $1 \mathrm{~mm}$ ) that crosses through the height of the sensor in the center of the gap as described in Fig.4.

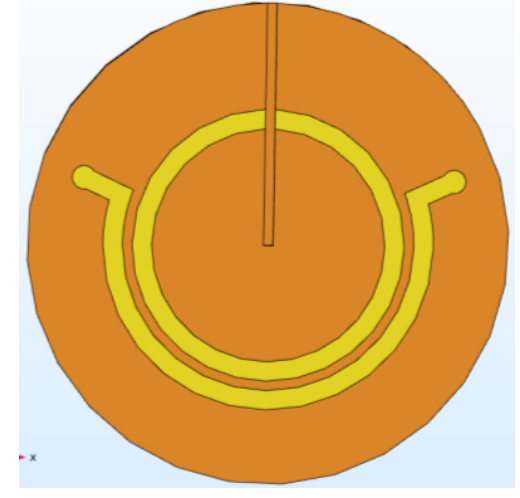

(a)

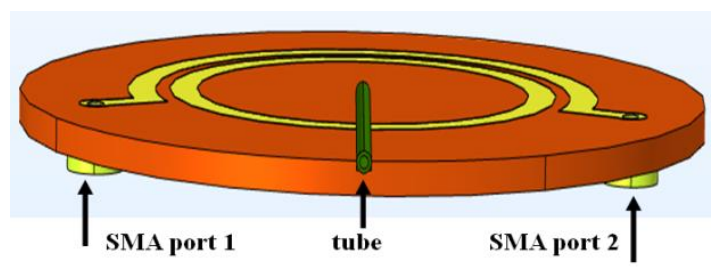

(b)

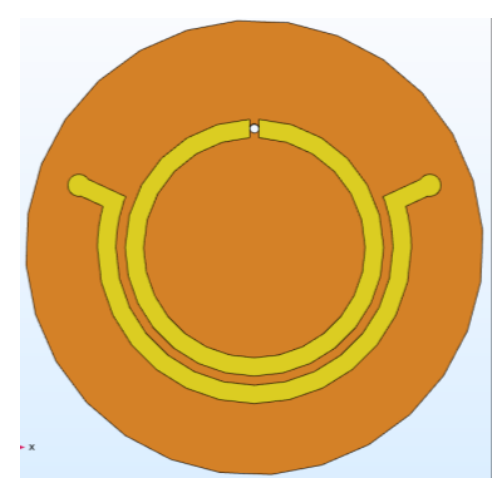

(c)

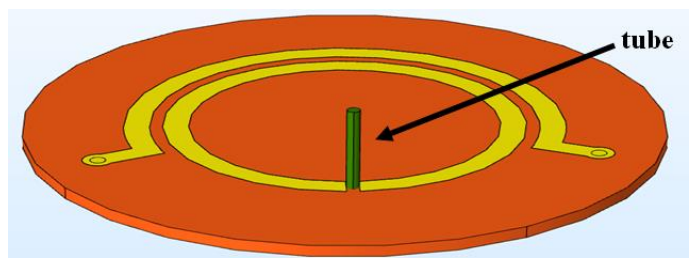

(d)

Figure 2. topologies of the microfluidic channel situation on microstrip SRR substrate (a) groove channel (b) with a tube fixed in a horizontal position, (c) a round hole (d) with a tube installed in a vertical position. 


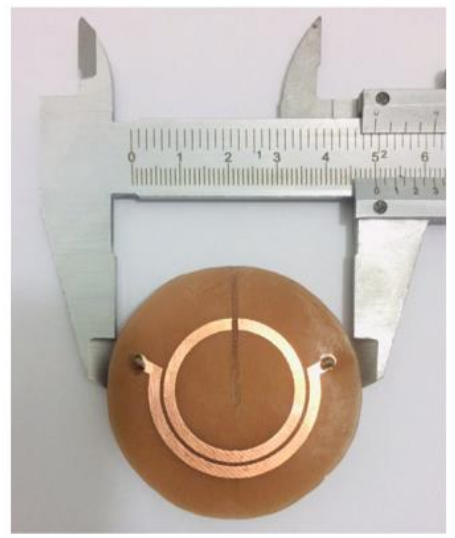

(a)

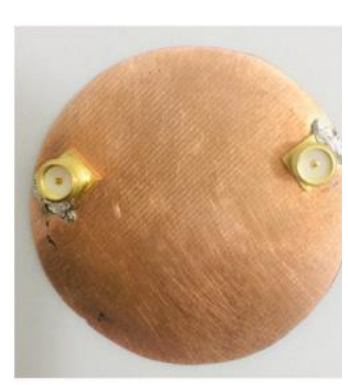

(b)
Figure 3. Photograph of the fabricated SRR sensor with SMA connectors. (a) Top view, (b) Bottom view.

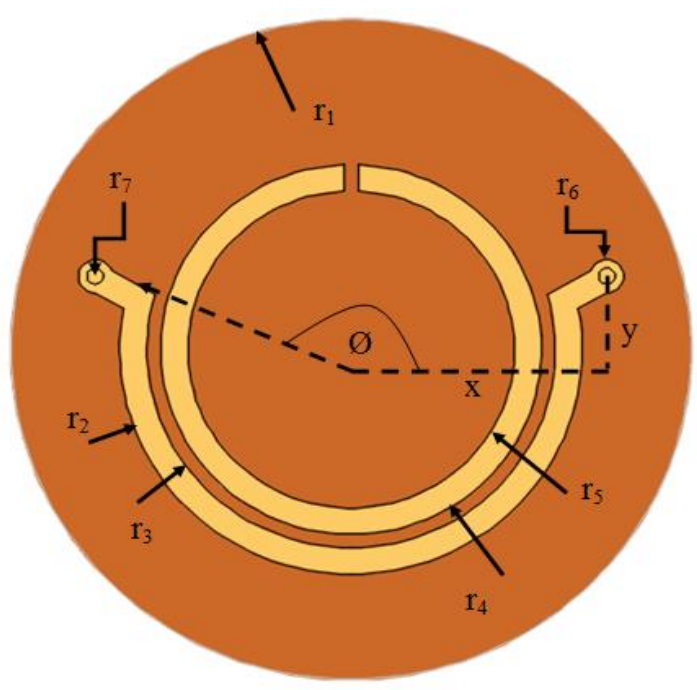

Figure 4. SSRR dimensions.

Table 1. Dimensions of sensor (mm)

\begin{tabular}{llllllllll}
\hline r1 & r2 & r3 & r4 & r5 & r6 & r7 & $\mathbf{x}$ & $\mathbf{y}$ & $\emptyset$ \\
\hline 25 & 17 & 15 & 14 & 12 & 1.2 & 0.65 & 19 & 5.4 & $164^{\circ}$ \\
\hline
\end{tabular}

Fig.5. Shows the system design used in this experiment, a quartz glass tube (outer diameter $1 \mathrm{~mm}$ and inner diameter $0.5 \mathrm{~mm}$ and relative permittivity 3.8) which has been used as a microfluidic channel carries liquid samples depends on capillary action so no need to syringe pump device. The sensor is packaged inside an aluminum cylindrical cavity (inner and outer diameter $50 \mathrm{~mm}, 70 \mathrm{~mm}$ respectively, and an internal height of $40 \mathrm{~mm}$ ) to achieve maximum quality factor by reducing dissipated loss.
At horizontal situation, the capillary is put in that groove and passes the cavity wall through the hole which gives the ability to fill the capillary from outside the cavity as shown in focus image in the Fig.5.(a) and the Fig.5.(b) is shown the horizontal position when the tube is inserted vertically into the hole at the gap and in which the electric field is parallel to the liquid filled in that tube. A portable vector network analyzer (VNA- KC901V) used to measure the change in the insertion loss and shift in frequency resonator of the sensor due to liquid perturbation.

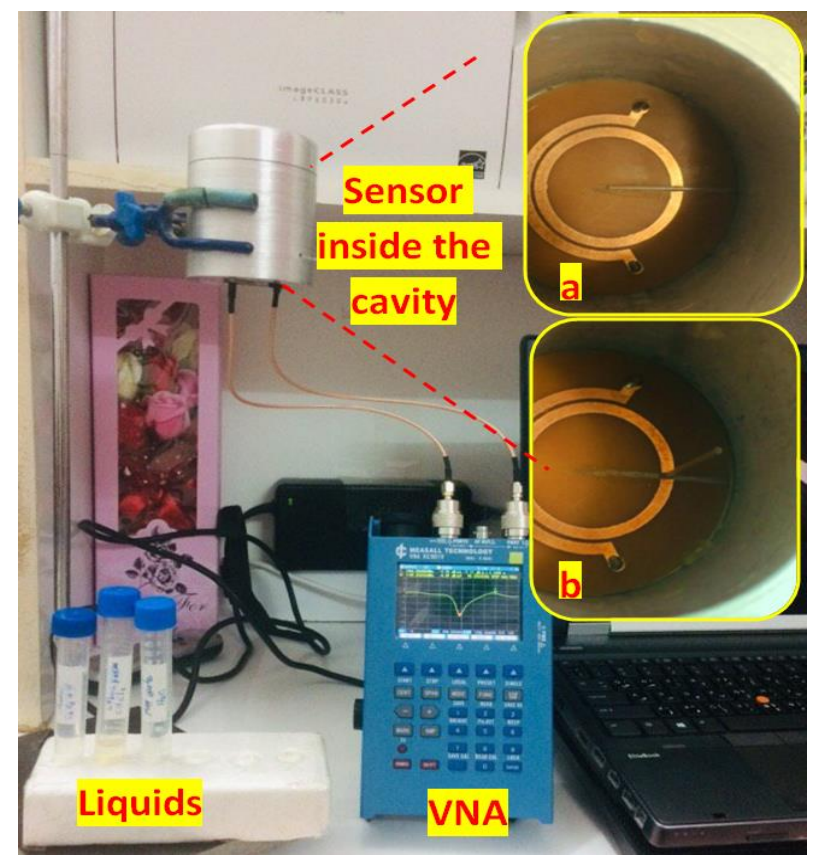

Figure 5. Experimental configuration with focus image for two tube position

\section{Measurement and Experimental Results}

A VNA screenshot as in Fig.6. shows the transmission coefficient $\left(S_{21}\right)$ was measured at first mode frequency resonator for both horizontal and vertical position of the capillary and when it was air-filled tube, and when it was filled with several solvents have tested such as water, ethanol, and chloroform and the results are shown in Fig.7. Table 2 lists all these measurements were taken at room temperature $25^{\circ} \mathrm{C}$, results show the extracted information from Fig.7. Such as the shift in resonant frequency which has been used to calculate complex permittivity using Debye's model equations ( $2 \& 3$ ) parameters of these equations are taken from [18].

Here, the measurement with an empty tube is considered as a reference to compute shift in resonance frequency and alter in loss of the sensor when the tube loaded with solvents. we show from the Table 2 shift in frequency decrease with decreasing dielectric constant $\left(\varepsilon^{\prime}\right)$ (give an approximately linear relationship) for both positions of the tube . 


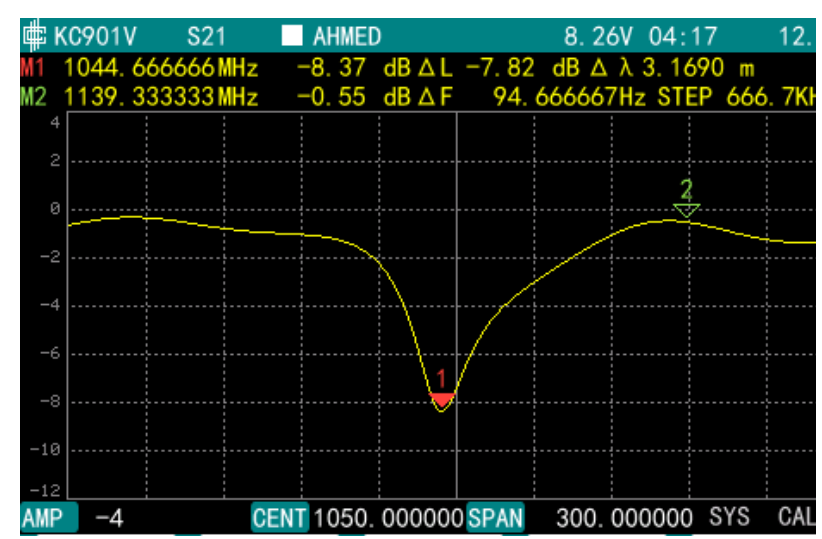

(a)

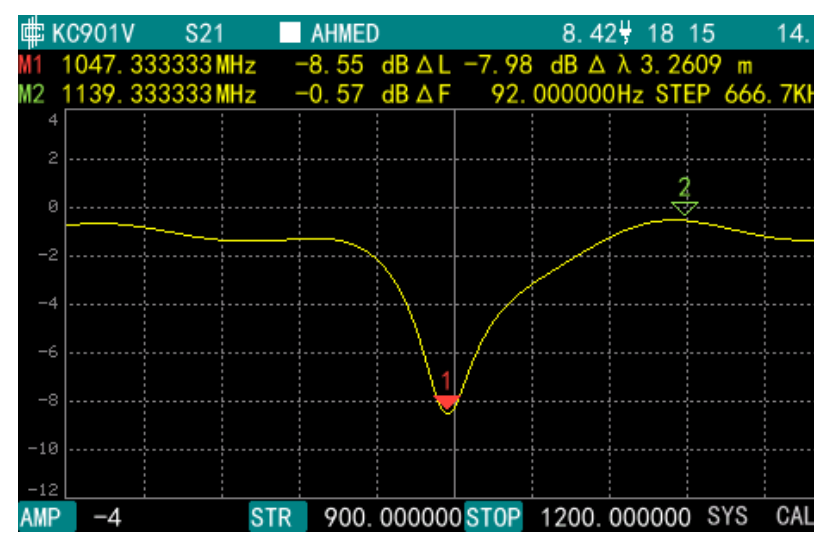

(b)

Figure 6. Mark 1 appears at the measured $S_{21}$ peak at the resonant frequency of the sensor when the empty quartz tube in its (a) vertical position (b) and horizontal position

The change reading between the measurements of the insertion loss (IL) of the sensor when the tube in the horizontal position are higher than that in the vertical position for the same sample due to the volume of the fluid exposed to the electric field is greater in another word, more energy absorbed by dipole of a solvent, and shift in the resonant frequency ( $\Delta \mathrm{fr}$ ) of the sensor in the vertical position are higher than that in the horizontal position with a rate of change $(2 \mathrm{MHz})$ due to the effective liquid volume (the effective length of the edge gap $(2 \mathrm{~mm})$ longer than the diameter of the tube $(1 \mathrm{~mm})$ ) is greater, which makes the sensor better sensitive than that in the vertical position and these results are summered in the Table 3.

Eventually, Table 4 states the characteristics of the current research work with a different type of pervious art sensor when a tube carried with chloroform.

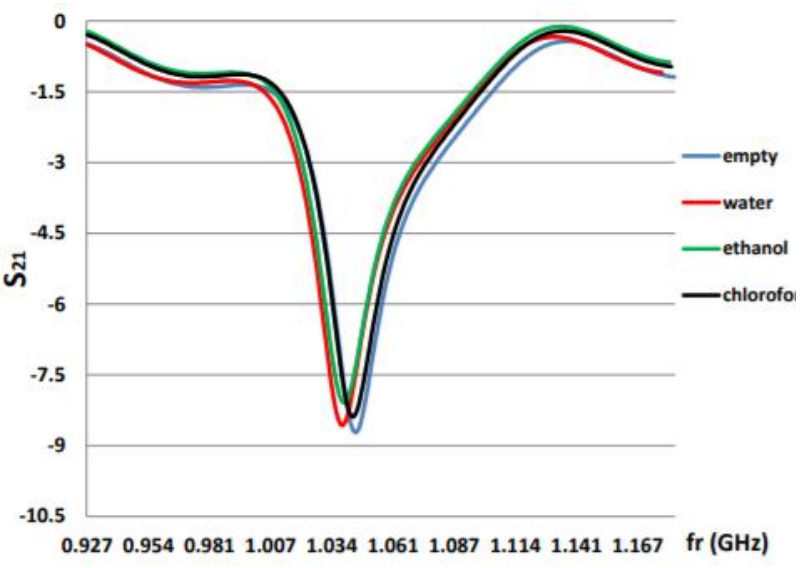

(a)

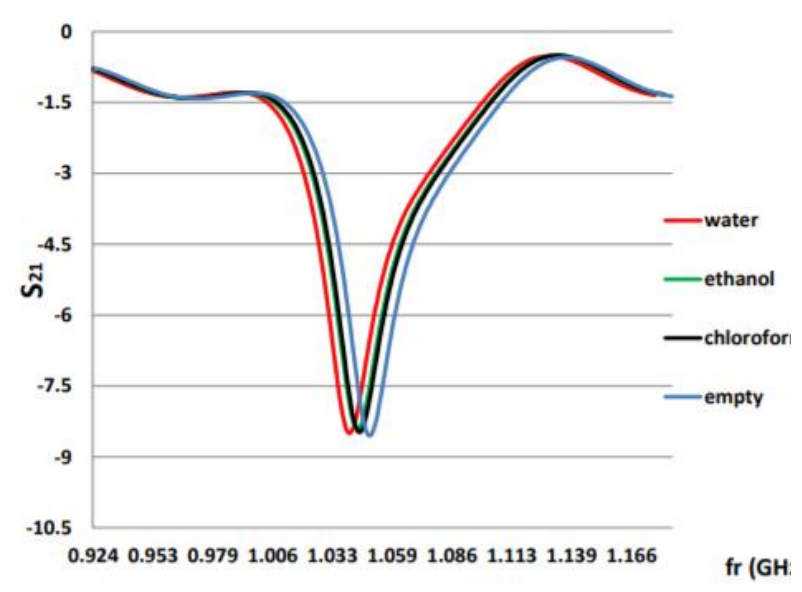

(b)

Figure 7. Measured $S_{21}$ of the sensor using quartz tube in its (a) vertical position (b) and horizontal position at resonant frequency

Table 3. Comparison of the results between the horizontal and the vertical position of the tube

\begin{tabular}{lcc}
\hline parameter & $\begin{array}{c}\text { Horizontal } \\
\text { position }\end{array}$ & $\begin{array}{c}\text { Vertical } \\
\text { position }\end{array}$ \\
\hline $\begin{array}{l}\text { Shift in the resonant } \\
\text { frequency }\end{array}$ & lower & higher \\
losses & higher & lower \\
sensitivity & low & better \\
\hline
\end{tabular}


Table 2. Measurement of complex permittivity of common fluids at the resonant frequency and compute the sensitivity

\begin{tabular}{|c|c|c|c|c|c|c|c|c|c|c|}
\hline \multirow{2}{*}{ liquid } & \multicolumn{5}{|c|}{ horizontal } & \multicolumn{5}{|c|}{ vertical } \\
\hline & fr (GHz) & IL (dB) & $\varepsilon^{\prime}-\mathrm{j} \varepsilon^{\prime \prime}$ & $\Delta \mathbf{f r}(\mathbf{M H z})$ & S (MHz) & fr (GHz) & IL (dB) & $\varepsilon^{\prime}-\mathrm{j} \varepsilon^{\prime \prime}$ & $\Delta \mathbf{f r}$ & S (MHz) \\
\hline empty & 1.0446 & 8.37 & 1 & 0 & 0 & 1.0473 & 8.55 & 1 & 0 & 0 \\
\hline water & 1.0377 & 8 & $78.187-\mathrm{j} 3.93$ & 6.9 & 0.09 & 1.04 & 8.5 & $78.18-3.94 \mathrm{j}$ & 7.3 & 0.1 \\
\hline ethanol & 1.04 & 7.62 & $13.626-\mathrm{j} 10$ & 4.6 & 0.36 & 1.0424 & 8.41 & $13.57-10.02 \mathrm{j}$ & 5 & 0.39 \\
\hline chloroform & 1.043 & 7.81 & $4.714-j 0.115$ & 1.6 & 0.43 & 1.045 & 8.48 & $4.713-0.115 \mathrm{j}$ & 2.3 & 0.62 \\
\hline
\end{tabular}

Table 4. A comparison of the proposed sensor with pervious work when a tube filled with chloroform at $25^{\circ} \mathrm{C}$

\begin{tabular}{|c|c|c|c|c|c|}
\hline Ref. & $\begin{array}{l}\text { Resonator } \\
\text { type }\end{array}$ & $\begin{array}{l}\text { substrate } \\
\text { type }\end{array}$ & Band type & $\begin{array}{l}\text { Active liquid } \\
\text { volume (nL) }\end{array}$ & $\begin{array}{l}\text { Sensitivity } \\
\text { (MHz) }\end{array}$ \\
\hline [3] & DSRR & Rogers 5880 & bandpass & 7 & 6.38 \\
\hline [6] & SRR & Rogers 5880 & bandpass & N/A & $<1.7$ \\
\hline [9] & DMS & Rogers 5870 & bandpass & 4500 & 1.3 \\
\hline [13] & SRR & Rogers 5870 & bandpass & 0.4 & 0.52 \\
\hline [21] & RMC & N/A & bandpass & 0.5 & 0.26 \\
\hline $\begin{array}{l}\text { present work } \\
\text { (vertical) }\end{array}$ & SRR & FR4 & stopband & 0.4 & 0.62 \\
\hline $\begin{array}{l}\text { present work } \\
\text { (horizontal) }\end{array}$ & SRR & FR4 & stopband & 0.23 & 0.43 \\
\hline
\end{tabular}

\section{CONCLUSIONS}

In summary, a new microwave microfluidic sensor was designed using a microstrip stop-band split-ring resonator with two different positions of the tube where the electric field is as large as possible over there. The sensor has been tested with several solvents to verify its sensitivity is to characterize liquid complex permittivity for both horizontal and vertical position of the tube and we found the loss of the sensor in the first position is higher than that in the second position but the last give noticeable change in the resonance frequency and hence the sensitivity is better.

\section{REFERENCES}

[1] M. Mode, S. MODE, S. MODE, Keysight Technologies, (2016).

[2] K. Saeed, R.D. Pollard, I.C. Hunter, Substrate integrated waveguide cavity resonators for complex permittivity characterization of materials, IEEE Transactions on Microwave Theory and Techniques, 56(10) (2008) 23402347.

[3] A.A. Abduljabar, D.J. Rowe, A. Porch, D.A. Barrow, Novel microwave microfluidic sensor using a microstrip split-ring resonator, IEEE Transactions on Microwave Theory and Techniques, 62(3) (2014) 679-688.

[4] A.A. Abduljabar, H. Hamzah, A. Porch, Double microstrip microfluidic sensor for temperature correction of liquid characterization, IEEE Microwave and Wireless Components Letters, 28(8) (2018) 735-737.

[5] A. Abduljabar, X. Yang, D. Barrow, A. Porch, Microstrip split ring resonator for microsphere detection and characterization, in: 2015 IEEE MTT-S International Microwave Symposium, IEEE, 2015, pp. 1-4.

[6] M. Abdolrazzaghi, M.H. Zarifi, M. Daneshmand, Sensitivity enhancement of split ring resonator based liquid sensors, in: 2016 IEEE SENSORS, IEEE, 2016, pp. 1-3.

[7] M.H. Zarifi, P. Shariaty, Z. Hashisho, M. Daneshmand, A non-contact microwave sensor for monitoring the interaction of zeolite $13 \mathrm{X}$ with $\mathrm{CO} 2$ and
CH4 in gaseous streams, Sensors and Actuators B: Chemical, 238 (2017) 1240-1247.

[8] M.H. Zarifi, M. Daneshmand, Monitoring solid particle deposition in lossy medium using planar resonator sensor, IEEE Sensors Journal, 17(23) (2017) 7981-7989.

[9] A.A. Abduljabar, N. Clark, J. Lees, A. Porch, Dual mode microwave microfluidic sensor for temperature variant liquid characterization, IEEE Transactions on Microwave Theory and Techniques, 65(7) (2017) 2572-2582.

[10] D.M. Pozar, Microwave Engineering, 4th ed., Wiley, 2012.

[11] W. Withayachumnankul, K. Jaruwongrungsee, A. Tuantranont, C. Fumeaux, D. Abbott, Metamaterial-based microfluidic sensor for dielectric characterization, Sensors and Actuators A: Physical, 189 (2013) 233-237.

[12] D.J. Rowe, S. Al-Malki, A.A. Abduljabar, A. Porch, D.A. Barrow, C.J. Allender, Improved split-ring resonator for microfluidic sensing, IEEE Transactions on microwave theory and techniques, 62(3) (2014) 689-699.

[13] A.A. Wahhab, A.A. Abduljabar, H.J. Albattat, High Sensitive Microwave Microfluidic Sensor Based on Split Ring Resonator for determination Liquid Permittivity Characterization, Al-Furat Journal of Innovation in Electronic and Computer Engineering, 1(01) (2020).

[14] K. Chang, L.-H. Hsieh, Microwave ring circuits and related structures, John Wiley \& Sons, 2004.

[15] R.G. Nitsche, J. Preissner, E.M. Biebl, A free space technique for measuring the complex permittivity and permeability in the millimeter wave range, in: 1994 IEEE MTT-S International Microwave Symposium Digest (Cat. No. 94CH3389-4), IEEE, 1994, pp. 1465-1468.

[16] K.C. Kao, Dielectric phenomena in solids, Elsevier, 2004.

[17] H. Frohlich, Theory of Dielectric, 2nd ed., Oxford Univ., New York, NY, USA, 1958.

[18] V.V. Komarov, Handbook of dielectric and thermal properties of materials at microwave frequencies, Artech house, 2012.

[19] P. Debye, Polar Molecules, The Chemical Catalog Company, Inc., New York, (1929) 77-108.

[20] F. Buckley, Tables of dielectric dispersion data for pure liquids and dilute solutions, Natl. Bur. Stand. Circ., 589 (1958) 7-8.

[21] H. Hamzah, A. Abduljabar, J. Lees, A. Porch, A compact microwave microfluidic sensor using a re-entrant cavity, Sensors, 18(3) (2018) 910. 